\title{
La potencialidad turística y sus oportunidades de emprendimiento. Caso Pasaje
}

\section{The tourism potential and its entrepreneurship opportunities. Passage case}

Carmen Arlene Lazo Serrano

Maria Isabel Bastidas Andrade

Freddy Ernesto Aguilar González

Melissa Paulina Calle Iñiguez

Universidad Técnica de Machala, Ecuador

Autor para correspondencia: clazo@utmachala.edu.ec

Fecha de recepción: 11 de Julio de 2017 - Fecha de aceptación: 10 de Agosto de 2017

Resumen: Este trabajo reúne tres pilares importantes, el turismo como generador de economías sostenibles, la potencialidad turística de un espacio geográfico y el emprendimiento asociado a los recursos naturales. Fueron estudiados en el cantón Pasaje provincia de El Oro, mediante una metodología analítica descriptiva no experimental. Para el levantamiento de la información se usaron las técnicas de: observación directa para el inventario y jerarquización de los recursos turísticos naturales, así como para la potencialidad turística; luego para identificar los emprendimientos adecuados a estas características se realizaron encuestas a los pobladores del cantón y focus group. Los resultados indican que existen 23 recursos turísticos naturales, a pesar de tener una buena accesibilidad no cuentan con el equipamiento necesario para la implementación de acciones turísticas inmediatas; sin embargo el recurso agua es el más importante y valorado por la población, existiendo una tendencia hacia el uso del mismo para emprendimientos turísticos, generando expectativas positivas en la zona para el desarrollo local.

Palabras claves: desarrollo sustentable; emprendimiento; potencialidad turística; recursos turísticos naturales

Abstract: This work brings together three important pillars, tourism as a generator of sustainable economies, tourism potential of a geographic space and entrepreneurship associated with natural resources. They were studied using a descriptive, non-experimental qualitative methodology. In order to collect information, the following techniques were used: direct observation for the inventory and hierarchization of natural tourism resources, as well as tourism potential; then to identify the enterprises were carried out surveys to the settlers of the canton and focus group. The results indicate that there are 23 natural tourism resources, despite having a good accessibility they do not have the necessary equipment for the implementation of immediate tourist actions; Nevertheless the water resource is the most important and valued by the population, there is a trend toward the use of water for tourism ventures, generating positive expectations in the area for local development.

Key words: sustainable development; entrepreneurship; touristic potentiality; natural tourism resources 


\section{Introducción}

La provincia de El Oro posee una geografía privilegiada que va desde el páramo hasta el manglar, con paisajes naturales excepcionales los recursos naturales se convierten en el principal atractivo para cualquiera que la visita. Pasaje hasta el 2015 según el Plan de Desarrollo y Ordenamiento Territorial (PDOT) cuenta con 81.897 habitantes en $451 \mathrm{~km} 2$, a $18 \mathrm{~km}$ de la capital Machala; con cuatro parroquias urbanas: Ochoa León, Loma de Franco, Tres Cerritos y Bolívar; y seis rurales, La Peaña, Cañaquemada, Uzhcurrumi, Casacay, El Progreso y Buenavista; es uno de los cantones con mayor población de la provincia. La característica principal de su geomorfología es determinada por las estribaciones de la Cordillera de los Andes y rodearse de relevantes ríos como el Jubones, Casacay, entre otros (Senplades, 2017).

\section{Recursos Naturales}

El PDOT del cantón Pasaje evidencia la existencia de recursos naturales muy llamativos y que son la base principal de este trabajo. Gary y Faundes (2013) definen a los recursos naturales como “...son todos los factores bióticos y abióticos de la naturaleza que puede utilizar el hombre para satisfacer sus necesidades", no obstante esta particularidad permite el uso de los recursos naturales, culturales y paisajísticos enfocados al ámbito turístico.

Tomando en consideración este distintivo se puede afirmar que si estos son gestionados de manera adecuada y ordenada, se puede llegar a un desarrollo sostenible a través de la generación e impulso de emprendimientos. El tema de sostenibilidad repunta con gran importancia desde una perspectiva económica, particularmente en Ecuador la presión ejercida por ser un país en vías de desarrollo, generando propuestas y estrategias que pretenden enfrentar las consecuencias de un aumento en la explotación de recursos naturales, propiciadas por la creciente población. Se puede señalar que la provisión de recursos naturales incide directa y positivamente en la generación de mejores condiciones de vida.

Además, los bienes y servicios que se extraen de los recursos proveen lazos identitarios, a través de su uso económico, religioso y cultural de un territorio en particular y su población; por ejemplo la minería, la pesca artesanal, la agricultura. En muchas ocasiones se convierten en espacios simbólicos y determinantes de un grupo de personas, dicho esto, los recursos naturales son esenciales en una nación y sociedad; de ellos dependen la salud, la alimentación, la cultura y supervivencia. Asimismo se puede definir que los recursos naturales turísticos tienen características particulares de uso del espacio con finalidades recreativas (Bertoni, 2005).

\section{Desarrollo sostenible y emprendimiento}

De acuerdo a los apartados anteriores, se evidencia una fuerte dependencia de la naturaleza y lo que nos ofrece, siendo necesario tomar medidas en ese sentido, surgiendo el término desarrollo sostenible.

Como antecedente se menciona que la importancia del desarrollo sostenible se basa en su relevancia social y difusión internacional, convirtiéndolo en un término controvertido en donde se diferencian dos tendencias claras. Para María de los Ángeles Murga (2013) el desarrollo 
sostenible primero ha de cumplir con ciertos parámetros y condiciones como por ejemplo: la vigilancia de la sociedad del sistema económico, la gestión de los recursos por parte de las comunidades locales y la reducción equitativa del consumo global; y, segundo condiciones sustentadas en términos de la óptica económica tradicional, en el que existe compatibilidad con el crecimiento permanente de un supuesto equilibrio ecológico a través de normas internacionales.

Existen problemáticas en la relación sociedad naturaleza, y entre actores turísticos, la sostenibilidad está orientada a la reducción de las mismas, enfocada en los factores: Económicos, Sociales, Políticos y Físico-naturales (Bertoni, 2005).

El desarrollo sostenible se afianza a través del Informe de Brundtland “...es aquel que satisface las necesidades actuales sin comprometer la capacidad de las generaciones futuras para satisfacer sus propias necesidades". Este concepto habla sobre solidaridad intrageneracional, solidaridad intergeneracional y necesidades, es decir todo apunta a un proceso de ampliación de las opciones de un ser humano: una vida saludable y larga, conocimientos, acceso a recursos para tener un nivel de vida adecuado (Xercavins, Cayuela, Cervantes, \& Sabater, 2005).

"El emprendimiento social es aquel que abarca las actividades y procesos llevados a cabo para descubrir, definir y explotar oportunidades con el fin de aumentar la riqueza social creando nuevas empresas o dirigiendo las organizaciones existentes de una manera innovadora" (Zahra, 2009). Lo importante es la creación de emprendimiento con carácter social más que económico, con la finalidad de dar solución a problemas de la comunidad en lugar de individuales. Se diferencia, de los emprendimientos pro-sostenibles al excluir objetivos orientados al mantenimiento de la naturaleza fuentes de apoyo de la vida y de la comunidad (Shepherd \& Patzelt, 2011).

El emprendimiento es esencial para el desarrollo de un país, es importante que los actores permitan generar iniciativas para romper las barreras y asegurar el éxito (Serrano, Riofrío, Brito, \& Sotomayor, 2016).

En un interesante trabajo de Medina, Medina y Gutiérrez (2016) mencionan la relación directa que existe entre los emprendimientos turísticos y el alivio de la pobreza, a través de la responsabilidad social. El estudio hace hincapié en los novedosos tipos de turismo y un término relativamente nuevo como el PPT pro-poor tourism y el CSR corporate social responsability; para ser efectivos deben apoyarse en políticas públicas para lograr proyectos en el territorio que tengan al menos estas características: (1) maximizar los ingresos del turismo para las comunidades locales, (2) la distribución equitativa entre la comunidad y (3) la generación de impactos turísticos positivos en los aspectos socioculturales y condiciones de pobreza.

También mencionan que la aplicación e implementación de programas de capacitación para el capital humano de una zona, tales como: educación básica y social, y mejoras en las capacidades y competencias en actividades turísticas como: acomodación, alimentos y bebidas, transporte, guianza turística, dirección y emprendimiento; mejorarían las capacidades de la población y aportarían al empoderamiento de las comunidades locales y la toma de decisiones. 
Dentro del desarrollo sostenible, la participación de las comunidades con emprendimientos orientados a mejoras sociales, es muy importante, de hecho el término de emprendimiento se afianza fuertemente en el siglo XXI y surge como respuesta a problemáticas de la sociedad con características más humanas, en donde el rol del emprendedor es vital así como las instituciones que respaldan las acciones y decisiones técnicas (Palacios, 2010), lo que concuerda fuertemente con los objetivos que persigue el desarrollo sostenible.

\section{Potencial turístico}

El potencial turístico de un territorio radica en su capacidad de atracción de turistas y satisfacción de sus necesidades, se establece la necesidad de dotar a los atractivos de instalaciones e infraestructuras necesarias para la realización actividades turísticas. Antes de iniciar cualquier actividad económica relacionada con el turismo es imperativo determinar su situación actual, a través de índices y factores claves para el desarrollo turístico; según Reyes y Sánchez (2005) los componentes que conforman la potencialidad de un determinado sitio son: los recursos, la accesibilidad y el equipamiento.

María de Lourdes Jarrín (2014) explica en su artículo Diagnóstico de la potencialidad de Tumbaco que las regiones, provincias, cantones y parroquias del Ecuador han crecido durante décadas de manera acelerada y la mayoría de una forma desordenada; es decir, no ha existido planificación en el territorio. Por consiguiente tratando de buscar soluciones a esta problemática, sin que cause tanto impacto al momento de incentivar el desarrollo de las poblaciones se señala al turismo como actividad que contiene estas características, más sin embargo no se lo abarca a profundidad.

Es preciso recalcar que un territorio por tener sitios naturales o culturales no significa que tienen potencial turístico, pues es condición que cuenten con características particulares como: conectividad, accesibilidad, infraestructura, entre otros, para que se facilite el desarrollo de planes, proyectos y programas.

Mientras el turismo avance, muchas más personas lo verán como una alternativa económica adecuada; diseñar y poner en marcha un pequeño negocio o emprendimiento turístico cada vez va tomando más fuerza. Este tema ha sido abordado por Oyarvide, Nazareno y Ferrales (2016) en un estudio realizado en la parroquia Tachina en la provincia de Esmeraldas, justifica la importancia del problema tomando en consideración que el turismo es una de las mayores industrias a nivel mundial, centrándose en el desarrollo sostenible; esto supone una gran oportunidad en algunas zonas urbanas y rurales en las que no existen otras alternativas de actividad económica, como en el caso de esta parroquia, y como parte del sector de los servicios, ofrece más oportunidades para el surgimiento de negocios locales que está compuesto mayoritariamente por Pymes.

Según Amer (2009) la consolidación de los logros de todo emprendimiento local necesita de actores estables en la intermediación con los agentes públicos y que existe un entendimiento tácito entre el gobierno y la empresa privada para su buen funcionamiento. 
Se identifica claramente que existe una necesidad de aprovechar las potencialidades del cantón Pasaje a través de emprendimientos para la generación de riqueza y mejora de la calidad de vida de sus habitantes. A simple vista existe poco aprovechamiento de los recursos naturales, por lo tanto es imperativo abordar el emprendimiento turístico como una herramienta para el desarrollo. En el trabajo de campo se identifican algunas ofertas turísticas incipientes, tomadas en consideración en el apartado de resultados.

\section{Implicaciones teóricas y prácticas del estudio}

Toda la revisión teórica de las relaciones entre la potencialidad de los recursos naturales, el desarrollo sostenible y el emprendimiento turístico, que se realiza en este estudio, aterriza en el planteamiento del siguiente cuestionamiento: ¿Existe potencialidad en los recursos turísticos naturales que impulse la creación de emprendimientos en el cantón Pasaje?

Con el propósito de dar respuesta a esta pregunta de investigación se plantean dos objetivos que deben ser respondidos:

- Determinar la potencialidad de los recursos turísticos naturales del cantón Pasaje.

- Establecer las opciones de emprendimientos que se desprenden de la potencialidad de los recursos turísticos naturales del cantón Pasaje.

En primera instancia se determinan la cantidad recursos turísticos naturales de todo el cantón Pasaje a través de un inventario aplicado la metodología del Ministerio de Turismo del Ecuador (MINTUR) y la potencialidad se estableció con la metodología de Reyes-Sánchez (2015); luego, para responder la segunda pregunta sobre las opciones de emprendimientos, se elaboró grupos focales y de la observación directa en el campo.

\section{Métodos}

El presente estudio forma parte del proyecto "Aprovechamiento de los Recursos Naturales de los Cantones Chilla y Pasaje para el Desarrollo Turístico Local”, del Grupo de Investigación 018 - El Oro Turístico de la Universidad Técnica de Machala.

En esta sección se describe la forma en que se va a recoger y analizar la información y las herramientas de investigación utilizadas. Se establece los métodos más efectivos para obtener la información necesaria para el análisis del problema. La metodología aplicada en este estudio es analítica descriptiva de carácter no experimental ${ }^{1}$, ya que se busca analizar las características turísticas de un territorio y determinar si es factible un desarrollo en este ámbito, para impulsar el emprendimiento luego de identificar las opciones; a través de estrategias descriptivas, cuantitativas y de observación.

El tipo de investigación realizada es analítico, cuantitativo, no experimental y de diseño transversal de causalidad; que concuerda con lo que se busca responder en las interrogantes. Los recursos turísticos naturales fueron el objeto de estudio, con ello se determinó la potencialidad turística natural de la zona a partir del inventario, jerarquización de MINTUR y potencialidad de

\footnotetext{
${ }^{1}$ Pretende observar fenómenos tal como se dan en su contexto natural, para posteriormente ser analizado
} 
los atractivos turísticos, fundamentándose en los lineamientos metodológicos sobre el potencial de los recursos turísticos naturales (Reyes Pérez \& Sánchez Crispín, 2005).

Adicional se aplicó una encuesta a la población para determinar usos y valoración de los recursos naturales y la predisposición de la población hacia los emprendimientos turísticos.

\section{Universo}

La población objetivo fueron los habitantes de Chilla y Pasaje, tomando en consideración la línea base del censo 2010 y proyectada para el 2016 de acuerdo a la tasa de crecimiento anual, esto es: Chilla 2.480 habitantes $(\mathrm{TC}=-0,34 \%)$ y Pasaje 83.079 habitantes $(\mathrm{TC}=2,00 \%)$, dando un total de población objetivo de 85.559 (INEC, 2017).

Tabla 1 Proyección crecimiento población 2016

\begin{tabular}{|c|c|c|c|}
\hline Cantón & Parroquia & Población & $\%$ \\
\hline \multirow[t]{4}{*}{ Chilla } & Chilla & 2.480 & 2,90 \\
\hline & Buenavista & 7.464 & 8,72 \\
\hline & Caña Quemada & 2.098 & 2,45 \\
\hline & Casacay & 2.804 & 3,28 \\
\hline \multirow[t]{5}{*}{ Pasaje } & La Peaña & 4.109 & 4,80 \\
\hline & Pasaje & 61.032 & 71,33 \\
\hline & Progreso & 4.527 & 5,29 \\
\hline & Uzhcurrumi & 1.045 & 1,22 \\
\hline & & 85.559 & 100,00 \\
\hline
\end{tabular}

Fuente: Inec: Censo 2010

Elaboración: Grupo De Investigación 018 "El Oro Turístico"

\section{Muestra}

Para el cálculo de la muestra se utilizó la fórmula de Pita Fernández, a partir del total de la población de Chilla y Pasaje, en la provincia de El Oro, así:

Donde:

$$
n=\frac{\mathrm{N} \cdot \mathrm{Z}^{2} \cdot \mathrm{p} \cdot(1-\mathrm{p})}{(\mathrm{N}-1) \cdot e^{2}+Z^{2} \cdot \mathrm{p} \cdot(1-\mathrm{p})}
$$

$\mathrm{n}=$ Tamaño de la muestra que queremos calcular

$\mathrm{N}=$ Tamaño del universo

$\mathrm{Z}=$ Valor del nivel de confianza, nivel de confianza $95 \% \rightarrow Z=1,96$

e = Límite aceptable de error muestral, e = 0,03 (3\%)

$\mathrm{p}=$ Proporción que esperamos encontrar.

Valor aproximado del parámetro que queremos medir, $\mathrm{p}=0,10(10 \%)$

Tabla 2 Cálculo Muestral

\begin{tabular}{ll}
\hline Total De La Población (N) & 85.559 \\
\hline Nivel De Confianza O Seguridad (1-A) & $95 \%$ \\
Precisión (D) & $3 \%$ \\
Proporción (Valor Aproximado Del Parámetro Que Queremos Medir) & $10 \%$ \\
Tamaño Muestral (N) & 382 \\
El Tamaño Muestral Ajustado A Pérdidas & \\
Proporción Esperada De Pérdidas (R) & $4,60 \%$ \\
\hline
\end{tabular}




\section{Muestra Ajustada A Las Pérdidas \\ 401 \\ Fuente: Inec: Censo 2010- Elaboración: Grupo De Investigación 018 "El Oro Turístico"}

La potencia estadística fue realizada en conjunto con el cálculo del tamaño de la muestra, trabajando con una potencia deseada mínima del $80 \%$; para dicha verificación se utilizó el software GPower v 3.1., del análisis se obtuvo $95 \%$ de potencia.

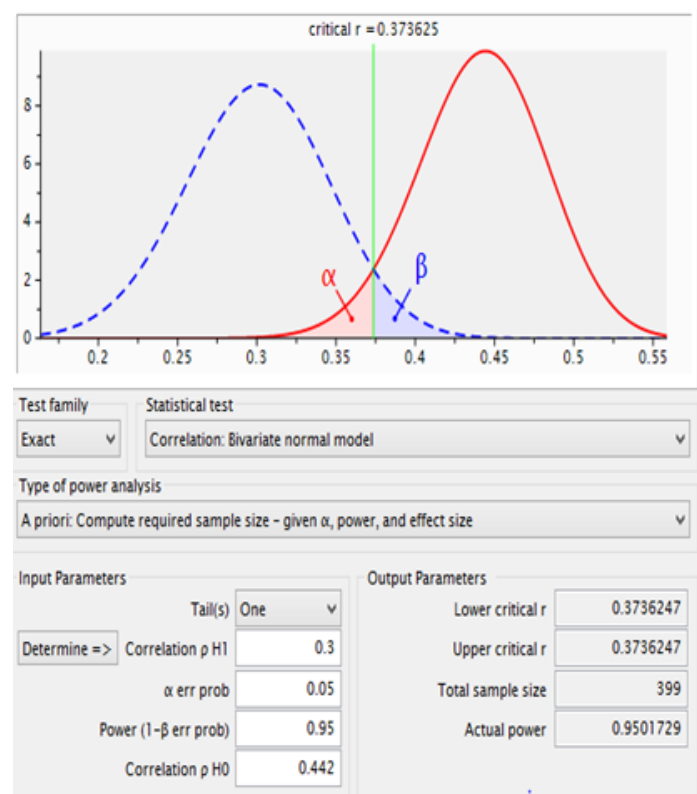

Ilustración 1 Potencia estadística

Fuente: Inec: Censo 2010

Elaboración: Grupo De Investigación 018 "El Oro Turístico"
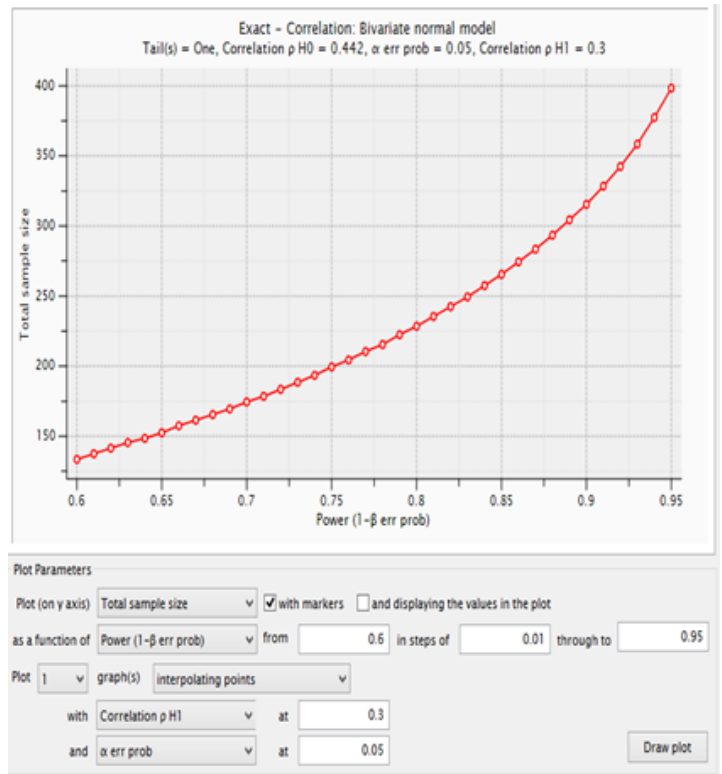

Ilustración 2 Parámetros de potencia y tamaño de muestra

Fuente: Inec: Censo 2010 Elaboración: Grupo De Investigación 018 “El Oro Turístico

\section{Muestreo:}

Se realizó un muestreo probabilístico de tipo aleatorio simple, se utilizó el sistema informático Microsoft Excel, el cual permitió seleccionar las localidades de acuerdo al porcentaje de población y determinar por parroquia el número de personas a encuestar; finalmente, con el número de individuos para la aplicación del instrumento metodológico de tipo encuesta. Para el caso de la potencialidad, el Universo objeto de estudio fueron los recursos turísticos naturales del cantón Chilla.

\section{Técnicas}

Se aplicó la encuesta a los pobladores de Chilla y Pasaje comprendidos en varios en grupos etarios, la misma que tuvo el carácter de anónima e incluyó: información de procedencia, edad, género, ocupación, instrucción, importancia de los recursos naturales, manejo de los recursos, usos de los recursos, valoración de los recursos, actividades económicas, comercialización y limitación de la localidad que condicionan el desarrollo turístico, con la finalidad de determinar el grado de valoración y uso de los recursos turísticos naturales. 
Asimismo se aplicó la técnica de la observación directa, en dos tipos de metodología, que permitió analizar y evaluar el potencial turístico natural de la localidad, usando: Metodología para el Inventario de Atractivos Turísticos, de la Gerencia Nacional de los Recursos Turísticos del Ministerio de Turismo del Ecuador (MINTUR). Con la finalidad de jerarquizar los atractivos turísticos naturales de la zona. Y la Metodología para determinar el potencial de los recursos turísticos naturales, en el Estado de Oaxaca, México de Reyes Pérez Oscar y Sánchez Crispín Álvaro, que permitió determinar el potencial turístico natural del lugar.

Finalmente se desarrollaron grupos focales en las zonas de estudio, con la finalidad de corroborar y profundizar los datos obtenidos en el trabajo de campo referente a los recursos naturales turísticos, y la predisposición de la población local para realizar emprendimientos como actividades de desarrollo local.

\section{Fase piloto del estudio}

\section{Procedimiento}

Se realizó una prueba piloto dirigida a 80 pobladores de cantones vecinos con características homogéneas o parecidas, a quienes se les aplicó la encuesta, previa presentación y consentimiento. Los datos obtenidos fueron sistematizados e ingresados en las hojas de cálculo del programa Microsoft Excel y luego ingresados o copiados en el programa SPSS. Se realizó un análisis factorial a través del alpha de Cronbach, obteniéndose un valor de 0.70 , que corresponde a una fiabilidad aceptable.

\section{Aplicación del instrumento:}

Una vez identificados y seleccionados se coordinó la aplicación del instrumento en las parroquias de los cantones involucrados según la distribución de la muestra por cada cantón y parroquia seleccionada aleatoriamente.

Para obtener el nivel jerárquico, se utilizó como instrumento de levantamiento de datos y medición la tabla anexa al documento Inventario de atractivos turísticos del MINTUR para luego obtener la potencialidad turística mediante el uso de fórmulas cuyos valores se basan en torno al tipo de factores que indica la metodología de Reyes y Sánchez (2005)

\section{Control de Calidad}

Los datos obtenidos se ingresaron progresivamente en el Microsoft Excel a la par como se iba recopilando la información, proceso realizado en el lapso de 1 mes; y, al finalizar el mismo se realizó el control de calidad de los datos.

\section{Equipo y materiales}

- Dispositivos de Geolocalización - GPS.

- Software informático "Microsoft Office" y programa SPSS versión 24.

- Cámara fotográfica 


\section{Análisis de datos:}

Los datos obtenidos en la encuesta fueron ingresados al programa Microsoft Excel, posterior a ello se exportaron al software estadístico SPSS versión 24, a través del cual se hizo el análisis estadístico utilizando el análisis descriptivo con un intervalo de confianza del 95\%, para los estadísticos de frecuencia, prueba de Chi cuadrado y valor de p para establecer la significancia estadística, la $\mathrm{V}$ de Crammer para establecer la magnitud del efecto y/o la fuerza de la dependencia, y el Odds Ratio (OR), esté último proceso fue donde se estableció si las variables dependiente e intervinientes eran factores de riesgo, protectores o son indiferentes.

Fue necesario transformar las variables politómicas a dicotómicas para poder determinar la relación existente, para ello se determinó con las respuestas obtenidas en la tabulación la sumatoria y posterior a ello el cálculo de los puntos de corte que nos permitirán codificar las respuestas transformadas o recodificadas, el detalle de lo expuesto se muestra a continuación:

Tabla 3 Valoración bienes y servicios: agua

\begin{tabular}{llll}
\hline Variables & $\begin{array}{l}\text { Punto De } \\
\text { Corte }\end{array}$ & Escala & Códigos \\
\hline Valoración Bienes Y Servicios: Agua & 20 & $<20$ & $\begin{array}{l}\text { Moderada A Baja Valoración Agua } \\
\text { Media A Alta Valoración Agua }\end{array}$ \\
\hline
\end{tabular}

Fuente: Trabajo De Campo 2016

Elaboración: Grupo De Investigación 018 "El Oro Turístico”

Para determinar la relación entre las variables de utilizó a más de Chi² el valor de "p", el cual permite que se acepte o rechace la potencial hipótesis o pregunta de investigación.

En lo referente a los datos de la tabla diagnóstica que corresponde a los atractivos turísticos naturales, se procedió a ingresar la información en una matriz de Excel, con el propósito analizar la información y cuantificar las ponderaciones de los elementos evaluados de los atractivos turísticos. El análisis se lo realizo evaluando ubicación, calidad, apoyo de los recursos turísticos naturales desde un valor intrínseco y valor extrínseco, lo que permitió tener como resultado nivel jerárquico de los atractivos turísticos naturales del cantón pasaje.

Finalmente para definir la potencialidad turística se utilizó los datos sobre nivel jerárquico y se evaluó estado natural del atractivo, accesibilidad y equipamiento de acuerdo a la metodología para determinar el potencial turístico.

\section{Inventario}

\section{Resultados}

El estudio permitió realizar una línea de base a macro escala considerando las 10 parroquias del cantón Pasaje las cuales se dividen en cuatro urbanas y seis rurales, cabe indicar que la mayoría de atractivos turísticos que cuentan con recursos naturales se encuentran distribuidos más en las parroquias rurales; para la generación de esta línea base se procedió a un estudio de campo para inventariar 23 atractivos con sus recursos naturales que, según la

\footnotetext{
2 Jerarquía I: LOCAL, cuyo factor está referido al grado de conocimiento del atractivo dentro del área municipal
} 
metodología de los documentos "Guía para el Levantamiento de atractivos turísticos del Ecuador" (2017) y "Metodología para el inventario de atractivos turísticos" - Gerencia nacional de recursos turísticos del MINTUR (MINTUR, 2017) fueron jerarquizados según sus características obteniendo un rango variable de I a III como se lo describe a continuación:

Tabla 4 Resumen de tipología y jerarquización

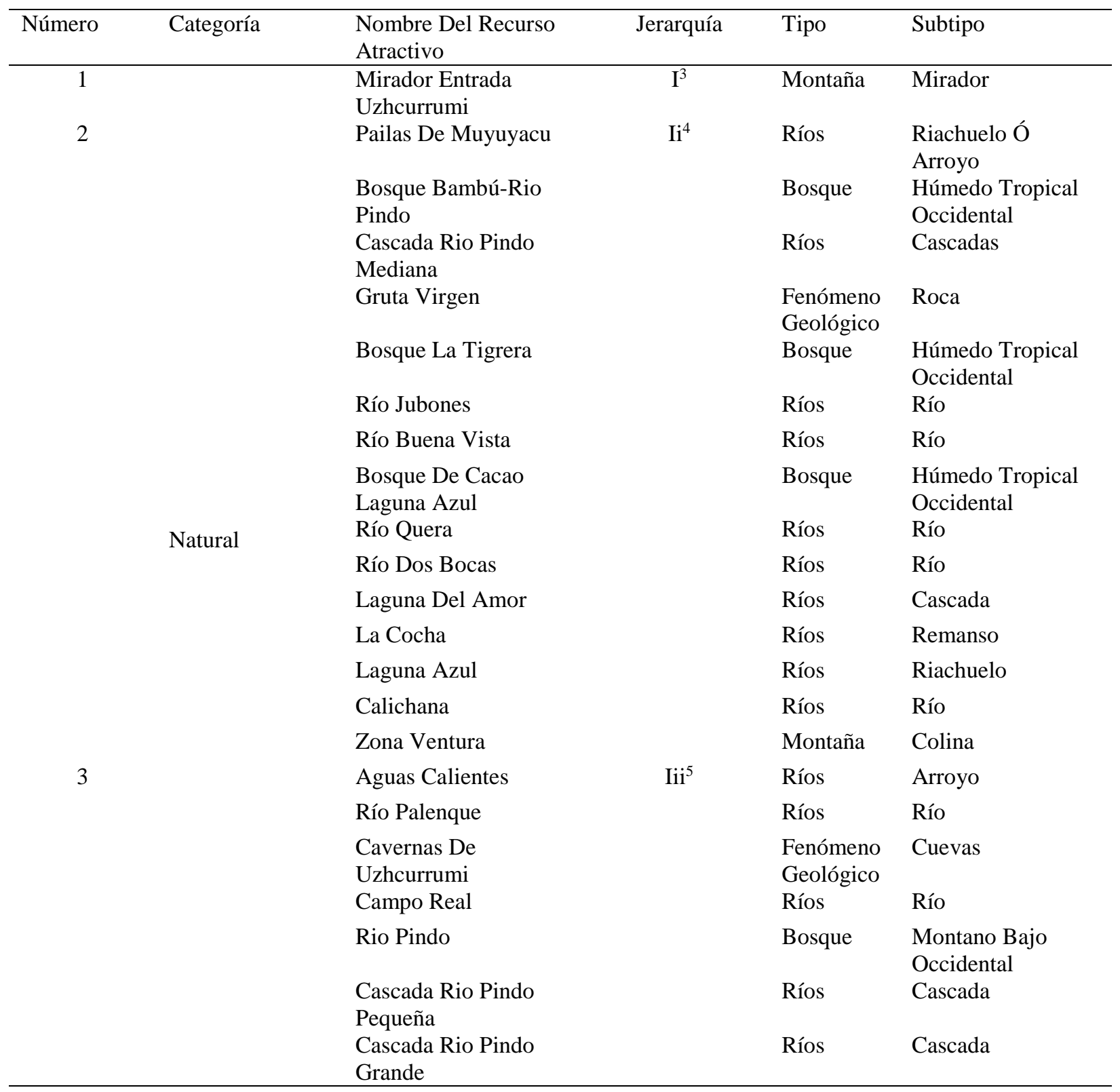

Fuente: Trabajo De Campo 2016 Elaboración: Grupo De Investigación 018 "El Oro Turístico"

\footnotetext{
${ }^{3}$ Jerarquía II: Provincial, donde el conocimiento o difusión abarca una o más provincias.

${ }^{4}$ Jerarquía III: Nacional, El Área De Difusión Abarca La Totalidad Del País.

${ }^{5}$ Jerarquía IV: Internacional, el atractivo supera las fronteras y es conocido en otros países, son generalmente los atractivos promocionados por el turismo receptivo
} 
Dentro de la metodología de inventario y jerarquización de atractivos turísticos del MINTUR la valoración de jerarquía I es la menor y de jerarquía IV es la mayor, los datos se levantaron mediante una ficha del MINTUR, instrumento que puede ser utilizado para el levantamiento de datos tanto de Sitios Naturales como de Manifestaciones Culturales, siendo la razón de esta investigación basada en el levantamiento de Sitios Naturales ${ }^{6}$.

Como se describe en la línea base obtenida, dentro del grupo de recursos levantados se encuentra que un atractivo tiene jerarquía I, quince atractivos tienen jerarquía II, y siete atractivos tienen jerarquía III.

En primer lugar el recurso natural agua forma una importante manifestación en este grupo. La mayoría de los recursos naturales levantados poseen una valoración de II lo cual es un indicador que son de interés provincial según la metodología aplicada, lo que sobresale dentro de este resultado de jerarquía II la mayoría de estos atractivos se basan en un recurso natural que es el agua cada uno de ellos con un tipo y subtipo que lo caracteriza.

En segundo lugar, está el grupo de atractivos con jerarquización III cuya valoración indica que poseen un tipo de interés nacional, indicando que también el recurso natural agua forma una importante manifestación en este grupo.

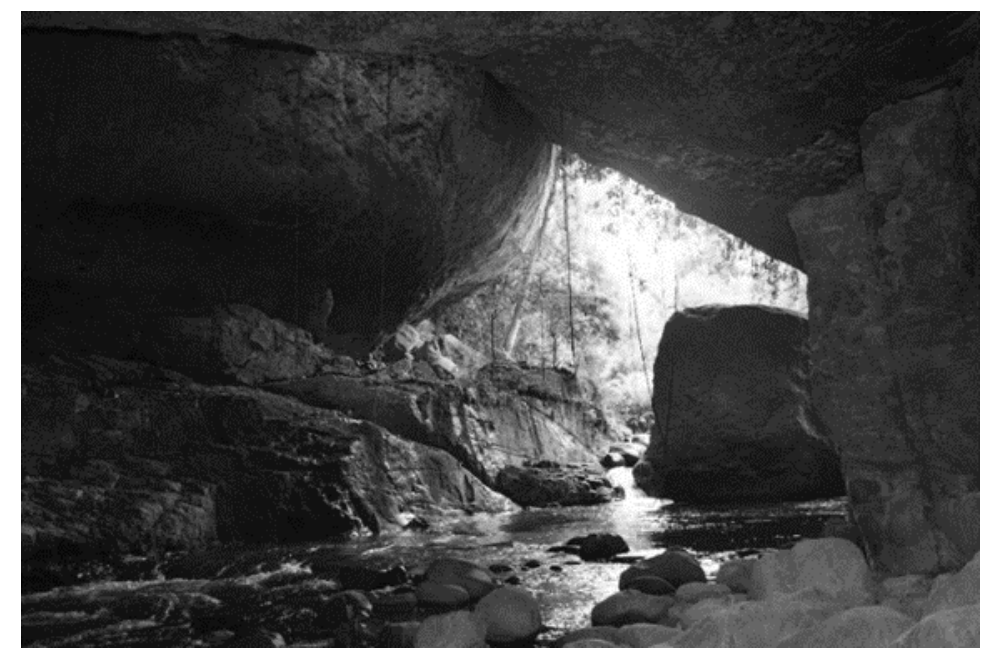

Ilustración 3 Cavernas de Uzhcurrumi-Jerarquía III

Fuente: Trabajo De Campo 2016

Elaboración: Grupo De Investigación 018 "El Oro Turístico"

En tercer lugar, con una jerarquía de I que es de importancia local, el cual pertenece al tipo montaña. En base al levantamiento y jerarquización de estos 23 atractivos turísticos se cita que el recurso natural agua forma una importante parte en la composición de este grupo por lo tanto se considera que la valoración de este recurso se lo determina en base a su uso y

\footnotetext{
${ }^{6}$ Las fichas contienen la siguiente estructura: Datos generales, número de ficha, encuestador, supervisor, evaluador, fecha, nombre del atractivo, categoría, tipo y subtipo, ubicación, geolocalización, calidad, valor intrínseco, altura, temperatura, precipitación, valor extrínseco, usos o simbolismo, estado de conservación del atractivo, estado de conservación del entorno, organización y cumplimiento, apoyo.
} 
aprovechamiento como tal en diversas actividades que pueden variar en el diario vivir, así como en las que se desarrollan de forma esporádica, todo ello depende de la edad de las personas que tienen accesibilidad a este recurso.

Conocido por su capacidad de generar efectos multiplicadores en la economía el sector turismo, también impulsa la generación de empleo directo e indirecto; estas condiciones han contribuido a dinamizar los mercados, sobre todo en las zonas rurales, y las actividades más practicadas en estos sectores son: turismo ecológico, turismo rural, turismo comunitario, turismo de aventura (Chen, 2005).

Otro aspecto que resalta dentro de los resultados es la definición del área de influencia ecológica, ya que lo natural juega un papel importante como recurso de la zona.

\section{Análisis y determinación del Potencial Turístico}

Debido a la cantidad de información recopilada, esta generó un amplio interés para su análisis y determinación de potencialidad turística y enfoque de emprendimientos, así como un estudio de las características de cada uno de los factores que forman parte de los atractivos y recursos naturales, para ello se aplicó la metodología aplicada por Reyes-Sánchez.

Se consideraron tres variables como factores de influencia circundante a cada uno de los sitios, estos factores son: Factor recurso.- los 23 que han sido levantados mediante trabajo de campo donde se incluyen geo formas, vegetación y elementos distintivos; Factor accesibilidad.donde destaca el transporte terrestre mediante vías de primer segundo y tercer orden, aquí se consideró también como facilidad de acceso la existencia de 1 aeropuerto nacional en el cantón de Santa Rosa a unos 20.6 km de distancia aproximadamente de la cabecera cantonal del cantón Pasaje cuyo nombre es el mismo.

Por último, el Factor equipamiento, cuyos datos actualizados hasta el mes de enero del año 2017 se determina que los sitios donde se encuentran geo localizados los atractivos y sus recursos naturales son carentes de equipamiento y facilidades turísticas, sin embargo, frente a ello la potencialidad de un desarrollo turístico de carácter sustentable y sostenible es viable.

Tabla 5 Variables utilizadas en el índice de potencialidad turístico natural en el Cantón

\begin{tabular}{cc} 
& Pasaje \\
\hline Factor Recurso (Fr) & Unidades Geomorfológicas \\
\cline { 2 - 2 } & Asociaciones Vegetales \\
Elementos Naturales Distintivos \\
Transporte Terrestre, Marítimo Y Aéreo
\end{tabular}

Factor Accesibilidad (Fa)

Gasolineras

Densidad Vial

Hoteles

Factor Equipamiento (Fe)

Establecimientos De Alimentos Y Bebidas 


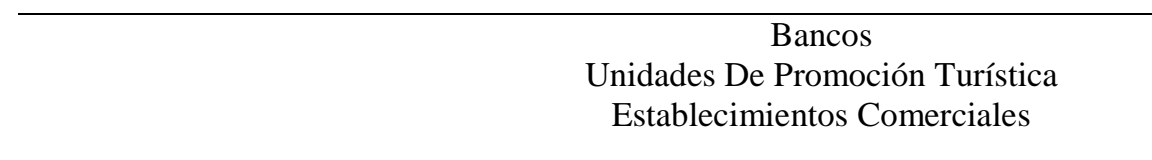

Fuente: (Reyes Pérez \& Sánchez Crispín, 2005)

Elaboración: Grupo De Investigación 018 "El Oro Turístico"

Los coeficientes de ponderación para las categorías de los atractivos turísticos con sus recursos naturales levantados suman en total 1. El autor Leno (1993, págs. 561-1204) propone que el valor turístico final de un recurso determinado está en función de su naturaleza e importancia, por lo mismo, este autor recomienda sumar una constante $\mathrm{K}=1$ para cada elemento:

\begin{tabular}{|c|c|c|}
\hline Recursos geomorfol & 0.20 & Recursos geomorfológicos $1+0.20$ \\
\hline Recurso vegetal & $=0.30$ & Recurso vegetal \\
\hline Elementos distintivos & $=0.50$ & Elementos distintivos \\
\hline
\end{tabular}

$$
1.00
$$

Una vez determinados estos parámetros se procedió a multiplicarlos con las jerarquías de la categoría de recurso correspondiente, los resultados obtenidos se encuentran en el siguiente cuadro. Los valores más elevados, corresponden a un alto potencial turístico, los índices más bajos se relacionan con los componentes que no son aprovechados para desarrollar esta actividad económica enfocada a diversas modalidades de emprendimientos.

\begin{tabular}{|c|c|c|c|}
\hline Jerarquías & $\begin{array}{l}\text { Geoformas } \\
(1.2)\end{array}$ & Vegetación (1.3) & $\begin{array}{l}\text { Elementos } \\
\text { naturales distintivos } \\
(1.5)\end{array}$ \\
\hline 1 & 1.2 & 1.3 & 1.5 \\
\hline 2 & 2.4 & 2.6 & 3.0 \\
\hline 3 & 3.6 & 3.9 & 4.5 \\
\hline 4 & 4.8 & 5.2 & 6.0 \\
\hline 5 & 6.0 & 6.5 & 7.5 \\
\hline
\end{tabular}

Fuente: (Reyes Pérez \& Sánchez Crispín, 2005)

Elaboración: Grupo De Investigación 018 "El Oro Turístico"

Una vez obtenida la ponderación de cada categoría, con sus elementos correspondientes jerarquías, se procedió a determinar el FR (factor recurso) de las seis parroquias rurales del Cantón Pasaje. La fórmula que se aplicó es la siguiente:

$\mathrm{FR}=\mathrm{R} 1+\mathrm{R} 2+\mathrm{R} 3$

Donde:

$\mathrm{FR}=$ factor recurso

$\mathrm{R} 1=$ geoformas

R2= vegetación

$\mathrm{R} 3=$ elementos naturales distintivos 
Identificados y levantados los 23 atractivos turísticos y sus recursos naturales del Cantón Pasaje se aplicó la formula multiplicando con su respectivo coeficiente de ponderación.

Tabla 7 Potencialidad Factor recurso

\begin{tabular}{clcc}
\hline $\mathrm{N}^{\circ}$ & Atractivo & Jerarquía & $\begin{array}{c}\text { Factor Recurso }\left(\sum \text { De R1, }\right. \\
\text { R2 Y R3 })\end{array}$ \\
\hline 1 & Pailas De Muyuyacu & II & 24.2 \\
2 & Aguas Calientes & III & 40.08 \\
3 & Rio Palenque & III & 31.80 \\
4 & Cavernas De Uzhcurrumi & III & 56.1 \\
5 & Campo Real & III & 15.6 \\
6 & Rio Pindo & III & 39.18 \\
7 & Bosque Bambú-Río Pindo & II & 16.2 \\
8 & Cascada Río Pindo Pequeña & III & 44.4 \\
& Cascada Río Pindo & II & 32 \\
9 & Mediana & & \\
10 & Cascada Río Pindo Grande & III & 60 \\
11 & Gruta Virgen & II & 27 \\
12 & Mirador Entrada & I & 16.01 \\
& Uzhcurrumi & & \\
13 & Bosque La Tigrera & II & 30 \\
14 & Río Jubones & II & 30 \\
15 & Río Buenavista & II & 16.2 \\
16 & Bosque De Cacao Laguna & II & 16.2 \\
& Azul & & \\
17 & Río Quera & II & 18.8 \\
18 & Río Dos Bocas & II & 31.8 \\
19 & Laguna Del Amor & II & 40 \\
20 & La Cocha & II & 21.6 \\
21 & Laguna Azul & II & 21.6 \\
22 & Calichana & II & 24.6 \\
23 & Zona Ventura & II & 40 \\
\hline & & &
\end{tabular}

Fuente: Trabajo De Campo 2016

Elaboración: Grupo De Investigación 018 "El Oro Turístico"

El segundo, Factor accesibilidad del territorio de estudio en el Cantón Pasaje fue valorado en base a la infraestructura de transporte terrestre, marítimo y aéreo disponible; el número de gasolineras y la densidad vial. La metodología que se utilizó en esta investigación para determinar el factor accesibilidad se explica a continuación:

Primero se jerarquizaron las categorías como se expresa en el siguiente cuadro:

Tabla 8 Componentes Factor Accesibilidad Y Jerarquía

\begin{tabular}{llcc}
\hline Jerarquías & Transporte (tipos) & $\begin{array}{c}\text { Gasolineras } \\
\text { (unidades) }\end{array}$ & $\begin{array}{c}\text { Densidad vial } \\
(\mathbf{k m} / \mathbf{k m 2})\end{array}$ \\
\hline 1 & Sin medios de transporte & 0 & 0 \\
2 & Marítimo & 0 & $0-29$ \\
3 & Aeródromos & 0 & $30-67$ \\
4 & Pasajeros & 3 & $68-150$ \\
\hline
\end{tabular}




\begin{tabular}{lccc}
\hline 5 & Aeropuerto & 1 & $150>$ \\
\hline & Fuente: Trabajo De Campo 2016 \\
& Elaboración: Grupo De Investigación 018 “El Oro Turístico”
\end{tabular}

La primera categoría diferencia el tipo de transporte. Su jerarquización otorga el valor máximo a los aeropuertos debido a que facilitan la llegada de turistas nacionales e internacionales, cabe indicar en este punto que en el Cantón Pasaje no cuenta con aeropuertos de transporte de turistas pero se considera como punto de referencia la existencia de un aeropuerto nacional ubicado en el Cantón Santa Rosa, la distancia entre las cabeceras cantonales de los cantones en mención es de 20.6 kms. Con un recorrido en automóvil de aproximadamente 31 minutos a $80 \mathrm{kms} / \mathrm{h}$; sitúa en un segundo nivel de importancia al transporte terrestre al ser que se moviliza a los visitantes hacia los diversos centros de recreación; coloca en tercer lugar a los aeródromos porque se encuentran en lugares de difícil acceso, pero estos pertenecen a las haciendas bananeras y cacaoteras de la zona; considera en cuarto sitio al transporte por agua porque es un servicio focalizado pero cabe indicar que no es un medio de transporte como tal utilizado esporádicamente para acciones de recreación como rafting o pesca artesanal por último, el nivel más bajo corresponde a las zonas que carecen de medios de transporte.

En este trabajo se consideró que el transporte de pasajeros tiene mayor relación con los visitantes, por lo tanto se le otorgó un valor de tres puntos, a las gasolineras, se los identificó como un servicio complementario y se les asignó dos puntos, a la densidad vial se la reconoció como parte de la infraestructura general de los territorios, fue considerada sólo con un punto de valoración.

Así, la fórmula empleada para determinar el FA de los 23 atractivos turísticos naturales del cantón Pasaje levantados quedó de la siguiente manera:

$\mathrm{FA}=3 \mathrm{~T}+2 \mathrm{G}+\mathrm{D}$

Donde:

$\mathrm{FA}=$ factor de accesibilidad

$\mathrm{T}=$ transporte

$\mathrm{G}=$ gasolineras

$\mathrm{D}=$ densidad vial

Por lo tanto, se multiplicaron los coeficientes de ponderación con los tipos y cantidad de servicios turísticos que tiene cada uno de los recursos naturales del Cantón los resultados son los siguientes:

Tabla 9 Potencialidad Factor Accesibilidad

\begin{tabular}{cccc}
\hline $\mathrm{N}^{\circ}$ & Atractivo & Jerarquía & Factor recurso $\left(\sum\right.$ de 3T, 2G y D) \\
\hline 1 & Pailas De Muyuyacu & II & 4211.36 \\
2 & Aguas Calientes & III & 115.9 \\
3 & Rio Palenque & III & 2808 \\
4 & Cavernas De Uzhcurrumi & III & 17550 \\
5 & Campo Real & III & 383.76 \\
6 & Rio Pindo & III & 6318 \\
7 & Bosque Bambú-Río Pindo & II & 193 \\
8 & Cascada Río Pindo Pequeña & III & 112.32 \\
9 & Cascada Río Pindo Mediana & II & 149.76 \\
\hline
\end{tabular}




\begin{tabular}{lccc}
\hline 10 & Cascada Río Pindo Grande & III & 292.5 \\
11 & Gruta Virgen & II & 84.24 \\
12 & Mirador Entrada Uzhcurrumi & I & 12519 \\
13 & Bosque La Tigrera & II & 292.5 \\
14 & Río Jubones & II & 45922.5 \\
15 & Río Buenavista & II & 1642.68 \\
16 & Bosque De Cacao Laguna Azul & II & 14.04 \\
17 & Río Quera & II & 1441.44 \\
18 & Río Dos Bocas & II & 19234.8 \\
19 & Laguna Del Amor & II & 292.5 \\
20 & La Cocha & II & 6612.84 \\
21 & Laguna Azul & II & 7132.32 \\
22 & Calichana & II & 8255.52 \\
23 & Zona Ventura & II & 37147.5 \\
\hline
\end{tabular}

Nota: $T=$ transporte, $G=$ gasolineras, $D=$ densidad vial.

Fuente: Trabajo De Campo 2016

Elaboración: Grupo De Investigación 018 "El Oro Turístico"

El tercero, Factor Equipamiento (FE), dentro de esta investigación refleja una característica nula puesto que cerca de los atractivos turísticos y sus recursos naturales no encontramos tipos de servicios que complementen la actividad turística como tal ya que todo está basado en un entorno natural, pero cabe indicar que en la cabecera cantonal si encontramos servicios complementarios como alojamiento, alimentos y bebidas, por citar dos de ellos.

Los resultados finales de la potencialidad son expuestos en la siguiente tabla:

Tabla 10 Tabla de resultados de potencialidad

\begin{tabular}{|c|c|c|c|c|c|c|}
\hline $\begin{array}{c}\text { Parroquias Del Cantón } \\
\text { Pasaje }\end{array}$ & Atractivo & Jerarquía & $\mathrm{Fr}$ & $\mathrm{Fa}$ & $\mathrm{Fe}$ & Total \\
\hline \multirow[t]{2}{*}{ Buenavista (Rural) } & Calichana & II & 24.6 & 8255.52 & 0 & 8280,12 \\
\hline & Río Buenavista & II & 16.2 & 1642.68 & 0 & 1658,88 \\
\hline \multirow{9}{*}{$\begin{array}{l}\text { Uzhcurrumi } \\
\text { (Rural) }\end{array}$} & Río Jubones & II & 30 & 45922.5 & 0 & 45952,5 \\
\hline & Cavernas De Uzhcurrumi & III & 56.1 & 17550 & 0 & 17606,1 \\
\hline & Río Pindo & III & 39.18 & 6318 & 0 & 6357,18 \\
\hline & Bosque De Bambú & II & 16.2 & 193 & 0 & 209,2 \\
\hline & Gruta De La Virgen & II & 27 & 84.24 & 0 & 111,24 \\
\hline & $\begin{array}{l}\text { Mirador Entrada } \\
\text { Uzhcurrumi }\end{array}$ & I & 16.01 & 12519 & 0 & 12535,01 \\
\hline & $\begin{array}{c}\text { Cascada De Río Pindo } \\
\text { Pequeña }\end{array}$ & III & 44.4 & 112.32 & 0 & 156,72 \\
\hline & $\begin{array}{c}\text { Cascada De Río Pindo } \\
\text { Mediana }\end{array}$ & II & 32 & 149.76 & 0 & 181,76 \\
\hline & $\begin{array}{c}\text { Cascada De Río Pindo } \\
\text { Grande }\end{array}$ & III & 60 & 292.5 & 0 & 352,25 \\
\hline \multirow{3}{*}{$\begin{array}{l}\text { El Progreso } \\
\text { (Rural) }\end{array}$} & Aguas Calientes & III & 40.08 & 115.9 & 0 & 155,98 \\
\hline & Pailas De Muyuyacu & II & 24.2 & 4211.36 & 0 & 4235,56 \\
\hline & Río Dos Bocas & II & 31.8 & 19234.8 & 0 & 19266,6 \\
\hline \multirow{5}{*}{$\begin{array}{l}\text { Casacay } \\
\text { (Rural) }\end{array}$} & Laguna Azul & II & 21.6 & 7132.32 & 0 & 7153,92 \\
\hline & La Cocha & II & 21.6 & 6612.84 & 0 & 6634,44 \\
\hline & Laguna Del Amor & II & 40 & 292.5 & 0 & 332,5 \\
\hline & Río Quera & II & 18.8 & 1441.44 & 0 & 1459,52 \\
\hline & $\begin{array}{l}\text { Bosque De Cacao } \\
\text { Laguna Azul }\end{array}$ & II & 16.2 & 14.04 & 0 & 30,24 \\
\hline
\end{tabular}




\begin{tabular}{|c|c|c|c|c|c|}
\hline & Bosque La Tigrera & 30 & 292.5 & 0 & 322,5 \\
\hline & Zonaventura & 40 & 37147.5 & 0 & 37187,5 \\
\hline $\begin{array}{l}\text { Cañaquemada } \\
\text { (Rural) }\end{array}$ & \multicolumn{5}{|c|}{$\begin{array}{c}\text { No Cuenta Con Atractivos Turísticos Que Incluyan Recursos Naturales Relevantes } \\
\text { Para Levantamiento. }\end{array}$} \\
\hline $\begin{array}{l}\text { La Peaña } \\
\text { (Rural) }\end{array}$ & \multicolumn{5}{|c|}{$\begin{array}{c}\text { No Cuenta Con Atractivos Turísticos Que Incluyan Recursos Naturales Relevantes } \\
\text { Para Levantamiento. }\end{array}$} \\
\hline Loma De Franco & Campo Real & 15.6 & 383.76 & 0 & 399,36 \\
\hline (Urbana) & Río Palenque & 31.80 & 2808 & 0 & 2839,8 \\
\hline $\begin{array}{l}\text { Ochoa León } \\
\text { (Urbana) }\end{array}$ & \multicolumn{5}{|c|}{$\begin{array}{c}\text { No cuenta con atractivos turísticos que incluyan recursos naturales relevantes para } \\
\text { levantamiento. }\end{array}$} \\
\hline $\begin{array}{l}\text { Bolívar } \\
\text { (Urbana) }\end{array}$ & \multicolumn{5}{|c|}{$\begin{array}{c}\text { No cuenta con atractivos turísticos que incluyan recursos naturales relevantes para } \\
\text { levantamiento. }\end{array}$} \\
\hline $\begin{array}{l}\text { Tres Cerritos } \\
\text { (Urbana) }\end{array}$ & \multicolumn{5}{|c|}{$\begin{array}{c}\text { No cuenta con atractivos turísticos que incluyan recursos naturales relevantes para } \\
\text { levantamiento. }\end{array}$} \\
\hline
\end{tabular}

El siguiente cuadro se identifica los niveles de potencialidad:

Tabla 11 Niveles de potencialidad

\begin{tabular}{ll}
\hline Niveles & Puntos \\
\hline I & $30,24-6600$ \\
II & $6600-13.200$ \\
III & $13.200-19800$ \\
IV & $19800-26.400$ \\
V & $26.400-33.000$ \\
VI & $33.000-39.600$ \\
VII & $39.600-45922.5$ \\
\hline \multicolumn{2}{c}{ Fuente: Trabajo De Campo 2016 }
\end{tabular}

Elaboración: Grupo De Investigación 018 "El Oro Turístico"

Los aspectos que resaltan de este proceso son: Inclusión de las comunidades aledañas para formar parte del uso y aprovechamiento dentro del sector turístico con opciones al desarrollo de modalidades de emprendimientos, siendo la comunidad del Uzhcurrumi la más opcionada para generar un desarrollo, así como también instituciones públicas con injerencia, y expertos del área del turismo.

\section{Valoración de los recursos naturales}

Para ser más precisos en la identificación de los recursos turísticos naturales, la potencialidad y el emprendimiento, el recurso que se consideró con mayor importancia en su valoración es el agua dado que este elemento está presente en la mayor parte de las parroquias investigadas generando a la vez niveles de potencialidad según su jerarquización y los factores de influencia.

A través de encuestas a los pobladores cercanos a los atractivos turísticos levantados se aplicó escalas de medición con suma precaución.

Tabla 12 Valoración Del Agua

\begin{tabular}{|c|c|c|c|c|c|c|}
\hline Variables & Valoración Del Agua & $\mathrm{Chi}^{2}$ & $\mathrm{P}$ & V. Cramer & Or & $\begin{array}{c}\text { Intervalo De } \\
\text { Confianza Al 95\% }\end{array}$ \\
\hline
\end{tabular}




\begin{tabular}{|c|c|c|c|c|c|c|c|}
\hline & $\begin{array}{c}\text { Moderado } \\
\text { A Bajo } \\
\text { Valoración } \\
\text { Del Agua }\end{array}$ & $\begin{array}{l}\text { Medio A } \\
\text { Alto } \\
\text { Valoración } \\
\text { Del Agua }\end{array}$ & Rango De Edad & & & $\begin{array}{l}\text { Límite } \\
\text { Inferior }\end{array}$ & $\begin{array}{l}\text { Límite } \\
\text { Superior }\end{array}$ \\
\hline $\begin{array}{l}\text { Adultos } \\
\text { Jóvenes }\end{array}$ & $60,60 \%$ & $39,40 \%$ & $\begin{array}{lll}6,332 & 1 & 0,012\end{array}$ & 0,126 & 2,259 & 1,191 & 4,674 \\
\hline $\begin{array}{l}\text { Adultos } \\
\text { Mayores }\end{array}$ & $39,50 \%$ & $60,50 \%$ & & & & & \\
\hline
\end{tabular}

Fuente: Trabajo De Campo 2016

Elaboración: Grupo De Investigación 018 "El Oro Turístico”

\section{Análisis e interpretación:}

En relación a la valoración del agua con los rangos de edad, la mayor proporción se corresponde al 60,60\% de los adultos jóvenes que valoran al recurso agua en la categoría de moderado a bajo en comparación con una proporción de 39,50\% de los adultos mayores que valoran en esta misma categoría al recurso; en cuanto a la asociación entre variables, existe una diferencia estadísticamente significativa al calcular el valor de Chi cuadrado de 6,332, mayor al valor de Chi cuadrado tabulado de 3,841, lo cual indica que existe relación estadística, valor de $\mathrm{p}$ $<0,05(0,012)$ que reafirma la relación entre variables; la fuerza de asociación entre las variables rango de edad y valoración del agua es "baja" (V de Cramer $=0,126)$; la valoración del agua desde la perspectiva de razón de oportunidades, el rango de edad constituye un factor de riesgo para la valoración del agua en los adultos jóvenes con un valor significativo de OR de 2,259 (IC al 95\%; LCi 1,191 - LCs 4,674), lo que se interpreta que los adultos jóvenes de las zonas involucradas en el proyecto tiene una valoración moderada a baja del recurso agua en 125,95\% más baja en consideración con aquellos adultos mayores con la misma categorización y criterios de valoración.

\section{Interés por actividades turísticas y recursos naturales}

Entre las actividades económicas de mayor interés, están el aprovechamiento de los recursos naturales para las actividades turísticas con un 30,42\%, otro cercano $26,18 \%$ para las actividades comerciales y de artesanías, 3,99\% servicios de alojamientos y bebidas.

\section{Discusión}

Este documento contribuye a los estudios sobre la relación entre el potencial de los recursos turísticos naturales y el impulso que esto genera para el interés y creación de emprendimientos, en correspondencia a tipos de turismo de naturaleza.

Determinar la potencialidad de los recursos turísticos naturales del cantón Pasaje fue una de las interrogantes planteadas desde el inicio de la investigación; durante este proceso de inventario y jerarquización, se identificaron 23 recursos turísticos naturales. Los resultados evidencian como Pasaje cuenta y en especial su parroquia rural Uzhcurrumi presenta una gran cantidad de este tipo de recursos, por ende todas las opciones mayormente potenciales van a girar en torno a ellos; con elementos distintivos como los ríos y lagunas, lo que concuerda con la 
investigación previa de Noblecilla, Ortíz, Ruiz, Encalada, Ordoñez (2016) en las zonas rurales de Pasaje, en donde se encuentran recursos naturales de gran potencial para hacer actividades de esta índole.

Los resultados de la aplicación del método de potencialidad, analizando $\mathrm{FR}^{7}, \mathrm{FA}^{8}$ y $\mathrm{FE}^{9}$; el factor recurso revela como la riqueza biogeográfica del Cantón Pasaje, se localiza, principalmente, en las zonas montañosas y circundantes a sus ríos, de esta manera evaluando los tractivos y determinando la potencialidad se ha dado una valoración natural y económica para el desarrollo de la actividad turística del Cantón Pasaje con el desarrollo de emprendimientos.

Otra interrogante planteada fue Establecer las opciones de emprendimientos que se desprenden de la potencialidad de los recursos turísticos naturales del cantón, los resultados que han surgido a partir de una encuesta y grupos focales son:

La edad del participante e involucrado en la presente investigación tiene una relación directamente proporcional con la valoración del recurso agua, esto es: a menor edad menor valoración del recurso, se tiene un enfoque "más" de explotación o extracción del recurso antes de conservación y/o protección.

La población local se encuentra desarrollando emprendimientos turísticos enfocados al aprovechamiento de los recursos naturales que tiene la zona de Uzhcurrumi; las actividades en las que se ha incursionado son los deportes de aventura tales como: rápel, rafting, káyak, trekking, ciclismo, y otras actividades complementarias como: canoppy, tarabita, camping, observación de paisajes, recorriendo senderos y riveras de los ríos del sector, entre otras. Actualmente este de emprendimientos ha sido incipiente y no existe un contacto directo y continuo con el territorio.

\section{Conclusiones}

A pesar de que la potencialidad turística de los recursos naturales del cantón Pasaje no es muy alta, no por la carencia de elementos que enriquecen el entorno natural, sino por la ausencia del FE (factor equipamiento), sin embargo, la accesibilidad y las características físicas de los recursos en sí, facilitan el desarrollo actividades enfocadas al ámbito turístico y la vinculación de las comunidades locales generando opciones para la incursión de emprendimientos.

Al hablar de potencialidad de recursos naturales, se puede mencionar que es un proceso de intervención y uso por parte del ser humano sobre los recursos naturales mediante la actividad turística y genera la modificación de un territorio específico, a la vez vincula a las comunidades locales y su cultura logrando un cambio de percepción del sitio por parte del visitante, así también una valoración del ambiente. Si se considera la incursión de turistas a visitar sitios naturales, existe la tendencia a mayor demanda esto puede generar un segmento de aplicación de emprendimientos en varias modalidades.

\footnotetext{
${ }^{7}$ Factor recurso (FR), metodología Reyes-Sánchez.

${ }^{8}$ Factor accesibilidad (FA), metodología Reyes-Sánchez.

${ }^{9}$ Factor equipamiento (FE), metodología Reyes-Sánchez.
} 
El desarrollo en una mayor escala del turismo de aventura del cantón Pasaje requiere de una serie de apoyo por parte de expertos en el área y la capacitación y concienciación por parte de los miembros de las comunidades involucradas para que esto genere un mejor desarrollo de las actividades que se pueden incursionar. Siguiendo los objetivos del mismo, se recomiendan tipos de turismo sostenible los cuales no comprometan los recursos naturales y culturales del territorio.

Esta investigación demuestra la existencia de potencialidad turística sobre el territorio del cantón Pasaje, de tal modo, se considera que si se aplican emprendimientos sólo por necesidad económica estos pueden tender al fracaso ya que guardan relación con la carencia de recursos financieros o la inexperiencia en la creación de negocios, consecuente a ello estaría la falta de visión empresarial de los emprendedores. Por ello, es necesario apoyarse con la ayuda de gobiernos locales planteando los tipos de emprendimientos en turismo a desarrollar por beneficio del incremento de la matriz productiva del cantón en mención y las comunidades involucradas; esto es recomendable en los casos donde abundan los sectores rurales con menor desarrollo y bajo crecimiento, sin dejar de lado la capacitación y actualización de conocimientos del capital humano.

\section{Bibliografía}

Álvarez, R., \& Leno, F. (1986). Estudio para la valoración económica. La Rioja: Instituto de Estudios Turísticos-Gobierno de La Rioja.

Amer, J. (2009). Emprendedores turísticos locales y su intermediación en la esfera de las políticas públicas: el caso de Mallorca. Una aproximación desde la sociología. . Pasos, 371-380. Recuperado de: http://www.pasosonline.org/Publicados/7309special/PS0309_3.pdf

Bertoni, M. (2005). Recursos Naturales en nodos turísticos. Aportes y Transferencias, 95-111. Recuperado de: http://www.redalyc.org/pdf/276/27690206.pdf

Chen, S. (2005). Turismo y Ambiente: un potencial para el desarrollo económico para Costa Rica. Revista Reflexiones, 25-37. Recuperado de: http://www.redalyc.org/articulo.oa?id=72920803002

Gary, C., \& Faundes, C. (2013). Recursos naturales: competencia, cooperación y conflicto en Sudamérica. Santiago: RIL.

INEC. (13 de junio de 2017). INEC. Obtenido de ecuadorencifras.gob.ec: http://www.ecuadorencifras.gob.ec/proyecciones-poblacionales/

Leno, F. (1993). La evaluación de los recursos turísticos. El caso del Canal Tomo II. Madrid: Editorial de la Universidad Complutense de Madrid.

Medina, D., Medina, R., \& Gutiérrez, F. (2016). A Sustainable Development Approach to Assessingthe Engagement of Tourism Enterprises in PovertyAlleviation. Sustainable Development, 220-236. 
MINTUR. (15 de junio de 2017). Guía para el levantamiento de atractivos turísticos del Ecuador. Obtenido de Portal Servicios MINTUR - Ministerio de Turismo: http://servicios.turismo.gob.ec/descargas/InventarioAtractivosTuristicos/anexoParte2_As ociacionEspacioTuristico_GuiaLevantamientoAtractivosTuristicos2017_2daEd.pdf

MINTUR. (15 de junio de 2017). Metodología para el inventario de atractivos turísticos. Obtenido de Portal Servicios MINTUR - Ministerio de Turismo: http://servicios.turismo.gob.ec/descargas/InventarioAtractivosTuristicos/Parte1_GuiaMet odologicaInventarioGeneracionEspacioTuristico2017_2daEd.pdf

Murga-Menoyo, M. (2013). Desarrollo sostenible. Problemáticas, agentes y estrategias. Madrid: McGraw-Hill/Interamericana de España S.A.

Noblecilla, M., Ortíz, L., Ruiz, J., Encalada, I., \& Ordoñez, Ó. (2016). Análisis del potencial turístico de las comunidades rurales. Caso de estudio: Comunidad Muyuyacu, El OroEcuador. Revista Interamericana de Ambiente y Turismo, 48-59. Recuperado de: http://riat.utalca.cl/index.php/test/article/view/322/pdf

Palacios, G. (2010). Emprendimiento social: integrando a los excluidos en el ámbito rural. Revista de Ciencias Sociales, 579-590. Recuperado de: http://www.redalyc.org/pdf/280/28016613002.pdf

Reyes Pérez, O., \& Sanchéz Crispín, Á. (2005). Metodología para determinar el potencial de los recursos turisticos naturalez en el Estado de Oaxaca. Cuadernos de Turismo (16), 153-173. Recuperado de: http://www.redalyc.org/pdf/398/39801609.pdf

Serrano, B., Riofrío, Ó., Brito, L., \& Sotomayor, G. (2016). apacidad Emprendedora Y Limitantes En La Generación De Emprendimientos: Una Mirada Desde La Condición De Género. European Scientific Journal, 96-107. Recuperado de: http://eujournal.org/index.php/esj/article/view/8359/7999

Shepherd, D., \& Patzelt, H. (2011). The New Field of Sustainable Entrepreneurship: Studying Entrepreneurial Action Linking "What Is to Be Sustained" With "What Is to Be Developed". Entrepreneurship Theory and Practice, 137-163. Recuperado de: doi:10.1111/j.1540-6520.2010.00426.x

Xercavins, J., Cayuela, D., Cervantes, G., \& Sabater, A. (2005). Desarrollo Sostenible. Barcelona: Ediciones UPC.

Zahra, S. (2009). How do threshold firma sutain corporate entrepreneurship? The role of the board and absorptive capacity. Journal of Business Venturing, 248-260. Recuperado de: http://www.firstorm.com/wp-content/uploads/2014/05/06How-do-threshold-firmssustain-corporate-entrepreneurship.pdf 